\title{
ARTICULAÇÃO COMPENSATÓRIA ASSOCIADA À FISSURA DE PALATO OU DISFUNÇÃO VELOFARÍNGEA: REVISÃO DE LITERATURA
}

\section{Compensatory articulation associated to cleft palate or velopharyngeal dysfunction: literature review}

\author{
Viviane Cristina de Castro Marino ${ }^{(1)}$, Jeniffer de Cássia Rillo Dutka ${ }^{(2)}$, \\ Maria Inês Pegoraro-Krook ${ }^{(3)}$, Aveliny Mantovan Lima-Gregio ${ }^{(4)}$
}

\section{RESUMO}

Tema: articulação compensatória na fissura palatina. Objetivo: contribuir para o aprofundamento de informações sobre os tipos de articulação compensatória descritos na literatura e, ainda, discutir as implicações e contribuições da avaliação clínica e instrumental na identificação destas produções. Conclusão: as articulações compensatórias merecem a atenção de clínicos e pesquisadores que atuam no Brasil, já que estas alterações são encontradas com grande freqüência em crianças e adultos com fissura palatina ou disfunção velofaríngea, o que compromete a qualidade de vida destes sujeitos. Os fonoaudiólogos devem aprofundar seus conhecimentos sobre os tipos de articulação compensatória e os procedimentos de avaliação, bem como devem estabelecer programas preventivos que favoreçam a aquisição fonológica sem o desenvolvimento dessas compensações.

DESCRITORES: Fissura Palatina; Fala; Distúrbios da Fala; Transtornos da Articulação

\section{INTRODUÇÃO}

A produção da fala de sujeitos com fissura de palato (FP) e/ou disfunção velofaríngea (DVF) é motivo de grande preocupação dos fonoaudiólogos que atuam na prevenção, na avaliação, no diagnóstico e na reabilitação das alterações na produção de fala associadas às malformações craniofaciais. Dentre as alterações de fala que podem ser

(1) Fonoaudióloga; Professor assistente doutor da Faculdade de Filosofia e Ciências da Universidade Estadual Paulista, UNESP; Marília, SP, Brasil; Doutor em Communication Sciences and Disorders pela Universidade da Flórida.

(2) Fonoaudióloga; Professor assistente doutor da Faculdade de Odontologia de Bauru da Universidade de São Paulo, USP; Bauru, SP, Brasil; Doutor em Communication Sciences and Disorders pela Universidade da Flórida.

(3) Fonoaudióloga; Professor titular da Faculdade de Odontologia de Bauru da Universidade de São Paulo, USP; Bauru, SP, Brasil; Doutor em Distúrbios da Comunicação Humana pela Universidade Federal de São Paulo.

(4) Fonoaudióloga; Doutor em Linguística pela Universidade Estadual de Campinas, UNICAMP; Campinas, SP, Brasil.

Conflito de interesses: inexistente encontradas nesta população, destacam-se as articulações compensatórias (AC) - também conhecidas como distúrbios articulatórios compensatórios - devido às implicações que estas podem acarretar na vida social, educacional e até mesmo profissional dos sujeitos.

As $A C$ são consideradas desvios na produção dos sons, que se estabelecem nas fases iniciais da aquisição fonológica, em decorrência de tentativas das crianças com alterações estruturais (FP e/ou DVF) para compensar funcionalmente o mecanismo velofaríngeo alterado ${ }^{1}$. Há consenso na literatura de que o problema estrutural traz modificações fisiológicas no sistema de produção da fala, levando ao desenvolvimento das $A C^{2}$. As $A C$ alteram o ponto articulatório, já que são produzidas em regiões do trato vocal que estão aquém do problema estrutural, ou seja, posterior à velofaringe ${ }^{3}$. Por essa razão, as $A C$, quando presentes, podem tornar a fala da criança ou do adulto ininteligível até mesmo para os familiares mais próximos, o que acaba comprometendo a qualidade de vida destes sujeitos de forma significante. Além disso, na presença de 
AC, a fonoterapia é essencial para o estabelecimento da produção padrão e, portanto, requer do profissional fonoaudiólogo amplo conhecimento sobre essas compensações, de forma a favorecer tanto sua identificação como o planejamento terapêutico apropriado. O fonoaudiólogo deve conhecer ainda as diferentes terminologias utilizadas pelos estudiosos para descrever os vários tipos de $\mathrm{AC}$, uma vez que diferenças terminológicas refletem o estado da arte no momento em que estas produções foram apresentadas na literatura. $O$ objetivo deste trabalho é contribuir para o aprofundamento de informações sobre os tipos de AC descritos na literatura e, ainda, discutir as implicações e contribuições da avaliação clínica e instrumental na identificação destas produções.

\section{MÉTODO}

A busca pelos trabalhos referenciados no presente artigo foi realizada por meio de uma combinação das palavras-chave, a saber: "fissura labiopalatina", "articulação compensatória", "disfunção velofaríngea", "análise acústica", "espectrografia", "nasoendosocopia", "videofluoroscopia", "eletropalatografia", "radiografia", bem como suas traduções para a língua inglesa. Os buscadores Scirus e Google Scholar serviram de apoio para o primeiro levantamento. Após separação e cruzamento dos dados apanhados nos buscadores, foram feitas buscas nas seguintes bases de dados: Medline, Scielo, Pubmed, Periódicos CAPES.

Foram utilizados livros, artigos completos, trabalho apresentado em evento, tese e monografia, em nível nacional e internacional. Os estudos que trazem as informações mais relevantes sobre os temas abordados neste artigo são reportados a partir da década de 1970. Os livros internacionais disponibilizaram grande parte das informações sobre as descrições clínicas das AC, já os artigos priorizaram a descrição instrumental destas produções compensatórias. Foram compiladas, ao todo, 60 referências, sendo 9 nacionais (7 diretamente relacionadas ao tema) e 51 internacionais (todas diretamente relacionadas ao tema).

\section{REVISÃO DE LITERATURA}

\section{Definição e Terminologia}

As AC são consideradas produções perceptivamente não aceitáveis, uma vez que não resultam em contraste nas consoantes da língua, sendo que os termos "ajuste compensatório" (compensatory adjustments) $)^{4}$ e "padrão alterado com substituição por sons laríngeos e faríngeos" (gross sound substitution error pattern - compensatory articulation $)^{2,5}$ são utilizados na literatura para se referir às estas produções. As AC são também definidas como constrições compensatórias que são "aprendidas" (compensatory misarticulations) durante a aquisição dos sons da fala, e que se distinguem das alterações de fala classificadas como obrigatórias (hipernasalidade, escape de ar nasal e fraca pressão) ${ }^{6,7}$. Outros autores classificam as AC como alterações de fala ativas em oposição às alterações de fala passivas (hipernasalidade e escape de ar nasal) ${ }^{8}$.

Em geral, as AC são consideradas produções atípicas caracterizadas pelo uso de pontos articulatórios laríngeos ou faríngeos em substituição às consoantes orais que requerem maior pressão intraoral ${ }^{4-7,9}$. As AC representam, então, o uso de modo articulatório, geralmente, preservado e de ponto articulatório posteriorizado ${ }^{6-7}$.

Alguns tipos de AC podem ser co-produzidas $^{1,6,10}$. Este tipo de produção também foi recentemente nomeado articulação dupla ${ }^{11}$, porém já foi erroneamente nomeado "coarticulação" (termo que se confunde com o fenômeno de coarticulação linguística). Na co-produção, a criança/adulto apresenta movimentos articulatórios simultaneamente em duas regiões do trato vocal, porém somente um modo de articulação ${ }^{1}$. Neste caso, o ponto articulatório não usual (compensatório) é o efetivo para gerar a produção enquanto o ponto articulatório envolvido no som alvo ocorre de forma simultânea ${ }^{7}$, porém não efetiva. Por exemplo, a oclusiva glotal pode ser co-produzida com movimentos simultâneos de lábios ao substituir a plosiva /p/ ou com movimento da língua ao substituir as plosivas /t/ e /k/. No entanto, a constrição do fluxo permanece na região laríngea, mais precisamente na glote ${ }^{12}$.

\section{Histórico}

As primeiras descrições referentes às constrições ou contatos atípicos realizados pela língua nas várias regiões do palato e da parede posterior da faringe em sujeitos com FP foram apresentadas inicialmente na década de 60 . Com base em estudos radiográficos, pesquisadore ${ }^{13}$ observaram uma tendência consistente de posteriorização dos pontos articulatórios que envolvem a língua nos sujeitos que apresentam FP.

$\mathrm{Na}$ década de 70 as primeiras descrições das AC propriamente dita restringiam-se a apenas dois tipos produções compensatórias: a oclusiva glotal e a fricativa faríngea ${ }^{4,14}$. Já na década de 80 , além destes dois últimos, outros três tipos de AC associados à fala de sujeitos com AC foram descritos radiograficamente ${ }^{6}$, a saber: plosiva faríngea, fricativa nasal posterior e plosiva dorso médio palatal. Além dessas produções, também foi reportada a 
fricativa velar ${ }^{6}$, como um tipo de produção compensatória previamente citada na literatura ${ }^{15}$. Com base em estudos nasendoscópicos e videofluoroscópicos, outro tipo de AC (a fricativa laríngea) ainda foi identificado, em $1985^{16}$.

Já na década de 90, constrições nasofaríngeas (previamente relatadas como fricativa nasal posterior $^{6}$ ) também foram observadas, a partir de estudos envolvendo eletropalatografia e espectografia $^{17}$. Nestes estudos, os autores observaram tais constrições tanto para as fricativas palatais quanto as africadas. Ainda observaram, a partir de medidas instrumentais, que movimentos similares àqueles usados para a produção da plosiva dorso médio palatal, também eram realizados quando os sujeitos por eles investigados produziam fricativas palatais e africadas. Em 1995, observa-se grande preocupação de uma autora ${ }^{18}$ em apresentar uma descrição que agrupasse os vários tipos de $A C$ relatados na literatura, bem como em apresentar descrições baseadas em avaliações instrumentais. Assim, além dos dez tipos de $A C$ reportados previamente na literatura (oclusiva glotal, fricativa laríngea, africada laríngea, plosiva faríngea, fricativa faríngea, africada faríngea, fricativa nasal posterior, plosiva dorso médio palatal, fricativa médio dorso palatal e africada médio dorso palatal), outros dois tipos de AC ainda foram reportados: a plosiva laríngea e a africada nasal posterior ${ }^{18}$ (Tabela 1). A autora, no entanto, não menciona a ocorrência de fricativa velar como um tipo de $\mathrm{AC}$ associado à fala de sujeitos com FP e/ou DVF.

Tabela 1 - Tipos de AC e suas respectivas substituições apresentados em $1995^{18}$

\begin{tabular}{ll}
\hline AC & CONSOANTES SUBSTITUÍDAS \\
\hline Oclusiva glotal (golpe de glote) & oclusivas (predominante) \\
\hline Plosiva laríngea & oclusivas \\
\hline Fricativa laríngea & fricativas \\
\hline Africada laríngea & africadas \\
\hline Plosiva faríngea & oclusivas \\
\hline Fricativa faríngea & fricativas \\
\hline Africada faríngea & africadas \\
\hline Fricativa nasal posterior & fricativas \\
\hline Africada nasal posterior & africadas \\
\hline Plosiva dorso médio palatal & oclusivas alveolares e velares \\
\hline Fricativa dorso médio palatal & fricativas \\
\hline Africada dorso médio palatal & africadas \\
\hline
\end{tabular}

Ainda em 1995, outra autora ${ }^{12}$ reportou os seis tipos mais comuns de AC (oclusiva glotal, fricativa faríngea, fricativa laríngea, plosiva faríngea, fricativa/ronco nasal e plosiva dorso médio palatal) associando-os ao problema anatômico ou fisiológico presente. A autora sugere que a plosiva dorso médio palatal pode ser observada na presença de fístula de palato e/ou anormalidades anatômicas mais severas enquanto a plosiva faríngea e fricativa faríngea podem estar presentes diante de uma DVF ou fístula. Segundo a autora, a oclusiva glotal, a fricativa laríngea e a fricativa nasal são associadas somente à DVF. Ainda enfatiza que, embora todos os tipos de AC sejam classificados como compensações, a origem destas compensações pode variar.

Em 1997, tendo como base sua experiência clínica, uma autora ${ }^{7}$ apresenta uma descrição detaIhada de sete tipos de AC, bem como os padrões comumente observados para cada tipo de AC (Tabela 2). Esta autora, no entanto, ressalta que não considerou a fricativa laríngea, descrita previamente como uma categoria distinta de $\mathrm{AC}^{16}$, mas sim, uma variação da fricativa faríngea. Além dos sete tipos distintos de $A C$, a autora descreve três padrões atípicos de produções posteriorizadas comumente observadas em sujeitos com fissura de palato e/ou DVF7 (Tabela 3). 
Tabela 2 - Tipos de AC e substituições descritos em $1997^{7}$

\begin{tabular}{ll}
\hline AC & CONSOANTES SUBSTITUÍDAS \\
\hline $\begin{array}{l}\text { Oclusiva glotal } \\
\text { (golpe de glote) }\end{array}$ & $\begin{array}{l}\text { oclusivas, fricativas e africadas } \\
\text { co-produção com consoantes de pressão }\end{array}$ \\
\hline Plosiva faríngea & $\begin{array}{l}\text { oclusivas velares } \\
\text { não ocorre co-produção }\end{array}$ \\
\hline $\begin{array}{l}\text { Fricativa faríngea } \\
\text { (fricativa laríngea) }\end{array}$ & $\begin{array}{l}\text { fricativas alveolares, palatais e africadas } \\
\text { co-produção com fricativas alveolares e palatais }\end{array}$ \\
\hline Africada faríngea & $\begin{array}{l}\text { africadas } \\
\text { não ocorre co-produção }\end{array}$ \\
\hline Fricativa velar & $\begin{array}{l}\text { oclusivas velares e fricativas alveolares } \\
\text { não ocorre co-produção }\end{array}$ \\
\hline Plosiva dorso médio palatal & $\begin{array}{l}\text { oclusivas alveolares e velares } \\
\text { não ocorre co-produção }\end{array}$ \\
\hline Fricativa nasal posterior & $\begin{array}{l}\text { fricativas alveolares, palatais e africadas } \\
\text { co-produção com consoantes de pressão }\end{array}$ \\
\hline
\end{tabular}

Tabela 3 - Produções posteriorizadas descritas em $1997^{7}$

\begin{tabular}{ll}
\hline POSTERIORIZAÇÕES & CONSOANTES SUBSTITUÍDAS \\
\hline Líquida (lateral) alveolar velarizada & líquida lateral alveolar \\
Líquida não lateral velarizada & líquida não lateral \\
Nasal alveolar velarizada & nasal alveolar \\
\hline
\end{tabular}

Em 2001, a literatura apresenta uma revisão histórica sobre as descrições das $A C$, resumindo essas descrições em oito tipos de produções compensatórias: oclusiva glotal, fricativa faríngea, fricativa laríngea, plosiva faríngea, africada faríngea, fricativa nasal posterior, fricativa velar, e plosiva dorso-médio palatal ${ }^{3}$. Nesta revisão, os autores apontaram que: (a) movimentos similares aos observados para a fricativa nasal posterior, descrita em 1981, também foram observados por outros autores durante a produção de africadas, plosivas e fricativas e (b) movimentos similares àqueles usados para a produção da plosiva dorso médio palatal também foram identificados quando os sujeitos investigados produziam fricativas palatais e africadas.

Em 2006, uma modificação na descrição original proposta previamente na literatura ${ }^{6,7}$ foi sugerida ${ }^{1}$. Em tal modificação, os autores ${ }^{1}$ incluem o que eles chamam de fricativa nasal como um tipo de articulação compensatória (Tabela 4) e passam a caracterizar a fricativa velar (anteriormente considerada como uma $\mathrm{AC}$ ) como um tipo de produção oral posteriorizada, além de apresentarem uma descrição detalhada sobre os demais padrões de produção posteriorizados por eles observados (Tabela 5).

Apesar de algumas divergências nas classificações das $A C$ sejam identificadas na literatura internacional consultada é evidente a grande preocupação por parte de clínicos e pesquisadores no sentido de se entender os vários tipos de ajustes articulatórios que são frequentemente observados nos sujeitos que apresentam FP e/ou DVF. No Brasil, informações sobre os tipos de AC também foram reportadas por vários autores ${ }^{19-24}$ que se preocuparam em apresentar estas informações derivadas da literatura internacional e/ou de sua própria experiência clínica. Em geral, estes relatos resumiram as produções mais comuns encontradas na população com FP e/ou DVF, incluindo a oclusiva glotal, a plosiva faríngea, plosiva dorso médio palatal, a fricativa faríngea, a fricativa nasal posterior, a fricativa velar e a fricativa nasal. A Tabela 6 apresenta os tipos de AC associados à FP/DVF e fistula, de acordo com as descrições/citações da literatura (nacional e internacional) consultada. 
Tabela 4 - Tipos de AC e substituições reportadas em $2006^{6}$

\begin{tabular}{ll}
\hline AC & SUBSTITUIÇÕES (Consoantes substituídas e co-produzidas) \\
\hline $\begin{array}{l}\text { Oclusiva glotal } \\
\text { (golpe de glote) }\end{array}$ & $\begin{array}{l}\text { oclusivas (predominante), fricativas e africadas } \\
\text { co-produção com consoantes de pressão }\end{array}$ \\
\hline Plosiva faríngea & $\begin{array}{l}\text { oclusivas velares } \\
\text { não ocorre co-produção }\end{array}$ \\
\hline $\begin{array}{l}\text { Fricativa faríngea } \\
\text { (fricativa laríngea) }\end{array}$ & $\begin{array}{l}\text { fricativas alveolares, palatais e africadas } \\
\text { co-produção com fricativas alveolares e palatais }\end{array}$ \\
\hline Africada faríngea & $\begin{array}{l}\text { africadas } \\
\text { não ocorre co-produção }\end{array}$ \\
\hline Fricativa nasal & $\begin{array}{l}\text { oclusivas (pode ocorrer) } \\
\text { fricativas alveolares e palatais (predominante) } \\
\text { não ocorre co-produção }\end{array}$ \\
\hline Plosiva dorso médio palatal & $\begin{array}{l}\text { oclusivas alveolares e velares } \\
\text { não ocorre co-produção }\end{array}$ \\
\hline Fricativa nasal posterior & $\begin{array}{l}\text { fricativas alveolares, palatais e africadas } \\
\text { co-produção com consoantes de pressão }\end{array}$ \\
\hline
\end{tabular}

Tabela 5 - Produções posteriorizadas reportadas em $2006^{6}$

\begin{tabular}{ll}
\hline POSTERIORIZAÇÕES & CONSOANTES SUBSTITUÍDAS \\
\hline Fricativa dorso médio palatal & fricativas sibilantes e africadas \\
Fricativa velar & fricativas sibilantes e africadas \\
Sonorantes alveolares velarizadas & nasal alveolar e líquida lateral alveolar \\
Sonorante velarizada ou uvular & líquida não lateral \\
\hline
\end{tabular}

\section{Variabilidade nas AC}

Na presença da FP e/ou de DVF (congênita ou pós-cirúrgica), a criança/adulto, como um regulatório, tenta encontrar outra área no trato vocal onde o fluxo e a pressão podem ser controlados ${ }^{38}$, gerando, então, oclusão ou fricção (por meio de um posicionamento posteriorizado da língua em direção à faringe ou ainda do posicionamento anormal da laringe e/ou epiglote). Nem todas as AC são produzidas como uma forma de compensar o funcionamento velofaríngeo deficiente. Existem compensações decorrentes de tentativas de minimizar as perdas de ar que podem ocorrer por meio das fístulas (palatais ou alveolares), como é o caso da plosiva dorso médio palatal. Além disso, existem compensações em que ocorre a participação do próprio véu palatino ou do MVF de forma a gerar fricção do ar, como ocorre na fricativa nasal posterior ou na fricativa velar. Observa-se, então, variabilidade nos tipos de AC.

\section{Tipos de AC}

A literatura consultada revela a existência de mais de 10 tipos de AC, conforme resume a Tabela 6. De forma geral, essas produções compensatórias envolvem movimentos constritivos realizados na laringe, faringe, no véu, no palato e/ou na cavidade nasofaríngea. Vários fatores podem justificar as divergências em relação à quantidade e à descrição das AC parecem estar relacionadas ao que os autores consideram como AC propriamente dita e o que eles classificam como "produções orais posteriorizadas"1,7. No caso das produções posteriorizadas, estas podem ocorrer para consoantes fricativas ou nasais/líquidas e, por definição, envolvem uma produção que se dá com o ponto articulatório posterior ao daquele esperado para a produção alvo, mas cuja produção ocorre ainda na cavidade oral, o que resulta em menor impacto na inteligibilidade de fala e, portanto, são mais aceitáveis por parte dos ouvintes. 
Tabela 6 - Tipos de AC associados à FP/DVF e fístula conforme a literatura consultada

\begin{tabular}{|c|c|}
\hline AC & DESCRIÇÃO/CITAÇÃO \\
\hline $\begin{array}{l}\text { Oclusiva glotal } \\
\text { (golpe de glote) }\end{array}$ & $\begin{array}{l}\text { Peterson-Falzone et al. }{ }^{1,3} \text {; } \text { Morley }^{4} \text {; } \text { Trost }^{6} \text {; } \text { Trost-Cardamone }^{7} \text {; } \\
\text { Hennigsson e Isberg }{ }^{10} \text {; Golding-Kushner }{ }^{12,25} \text {; Morris }{ }^{14} \text {; } \text { Bzoch }^{15} \text {; Witzel } \\
\text { Mattos }\end{array}$ \\
\hline Plosiva laríngea & Witzel $^{18}$ \\
\hline Fricativa laríngea & 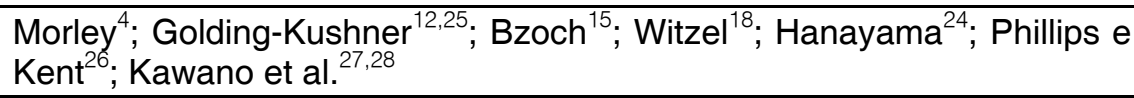 \\
\hline Africada laríngea & Witzel $^{18} ;$ Kawano et al. ${ }^{27-28}$ \\
\hline Plosiva faríngea & $\begin{array}{l}\text { Peterson-Falzone et al. }{ }^{1} \text {; } \text { Trost }^{6} \text {; } \text { Trost-Cardamone }^{7} \text {; } \text { Witzel }^{18} \text {; Mattos }{ }^{19} \text {; } \\
\text { Vicente e Buchala }{ }^{20} \text {; Altmann et al. }{ }^{21} \text {; Jesus et al. }{ }^{22} \text {; Genaro et al. }{ }^{23} \text {; } \\
\text { Hanayama }{ }^{24} \text {; Phillips e Kent }{ }^{26} \text {; Kawano et al. }{ }^{28} \text {; Kummer }{ }^{31} \text {; Honjow e } \\
\text { Isshiki }^{33}\end{array}$ \\
\hline Fricativa faríngea & 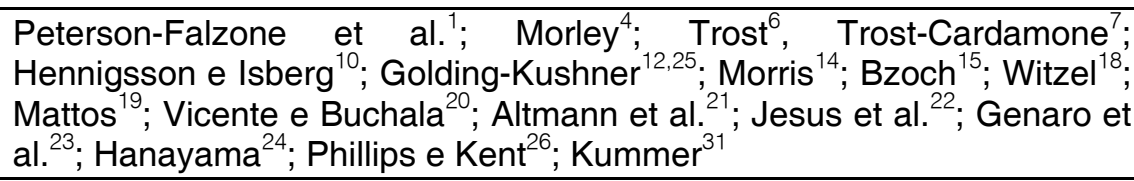 \\
\hline Africada faríngea & 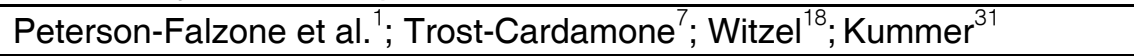 \\
\hline Fricativa velar & $\begin{array}{l}\text { Trost }^{6} ; \text { Trost-Cardamone } \\
\text { Jesus et al. }{ }^{22} \text {; } \text { Bzonaro et al. }^{23} ;{\text { Phillips e } \text { Kent }^{12} ; \text { Kummer }^{31}}^{19} \text { e } \text { Kuchala }^{20} \text {; }\end{array}$ \\
\hline Fricativa nasal posterior & $\begin{array}{l}\text { Peterson-Falzone et al. }{ }^{1} \text {; } \text { Trost }^{6} ; \text { Trost-Cardamone } \\
\text { Michi }\end{array}$ \\
\hline Africada nasal posterior & Witzel $^{18}$ \\
\hline Fricativa nasal & $\begin{array}{l}\text { Peterson-Falzone et al. }{ }^{1} \text {; Harding e Grunwell }{ }^{8} \text {; Altmann et al. }{ }^{21} \text {; Phillips e } \\
\text { Kent }^{26} \text {; Sell et al }\end{array}$ \\
\hline Plosiva dorso médio palatal & 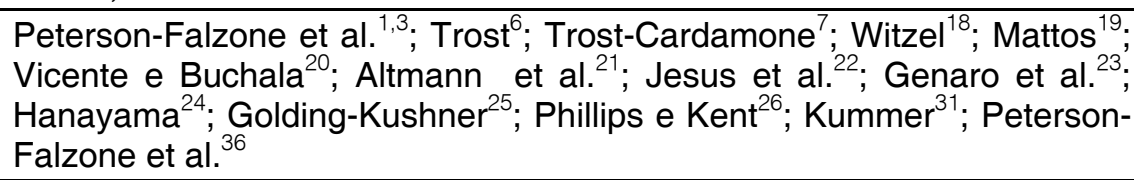 \\
\hline Fricativa dorso médio palatal & $\begin{array}{l}\text { McWilliams et al. }{ }^{9} \text {; Yamashita e Michi }{ }^{17} \text {; Witzel }{ }^{18} \text {; Phillips e } \text { Kent }^{26} \text {; Hoch } \\
\text { et } \text { al }^{37}\end{array}$ \\
\hline Africada dorso médio palatal & Yamashita e Michi $^{17}$; Witzel ${ }^{18}$ \\
\hline
\end{tabular}

Além disso, deve-se considerar que os procedimentos utilizados para a identificação das AC podem influenciar em sua classificação. Ainda que vários tipos de $\mathrm{AC}$ sejam identificados por meio de julgamentos perceptivo-auditivos associados à descrição dos movimentos articulatórios envolvidos em tais produções $4,6,7,15$ é inegável a contribuição da avaliação instrumental na identificação e caracterização das AC (Tabela 7). As avaliações instrumentais podem favorecer a identificação de ajustes articulatórios compensatórios que podem não ser facilmente percebidos por meio de avaliação clínica, o que resulta em maior variabilidade de descrições. Assim, a documentação destas produções obtidas por meio de avaliação radiográfica'; análise acústica ${ }^{16,17,26,33,39,40}$, videofluoroscopia e/ou nasoendoscopia $^{16,18}$ e eletropalatografia ${ }^{17,41-43}$ confirmaram descrições prévias baseadas em julgamentos perceptivo-auditivos das AC associados à $\mathrm{FP}$ e/ou DVF, bem como revelaram estratégias articulatórias até então não descritas na literatura. Por outro lado, as divergências na classificação das AC bem como no uso ou não de recursos instrumentais podem explicar porque enquanto um autor ${ }^{7}$ descreve a existência de sete tipos distintos de $A C$, outros referem oito ${ }^{26}$ ou até mesmo dez tipos ${ }^{18}$ distintos de produções compensatórias. 
Tabela 7 - Instrumentos objetivos de avaliação utilizados na identificação de cada tipo de AC

\begin{tabular}{|c|c|c|}
\hline AC & INSTRUMENTO & AUTOR \\
\hline \multirow{3}{*}{$\begin{array}{l}\text { Oclusiva glotal } \\
\text { (golpe de glote) }\end{array}$} & Videofluoroscopia & Kawano et al. $^{28}$ \\
\hline & Nasoendoscopia & Witzel $^{18} ;$ Kawano et al. ${ }^{27,28} ;$ Kido et al. $^{29,30}$ \\
\hline & Espectrografia & $\begin{array}{l}\text { Phillips e Kent }{ }^{26} \text {; Kawano et al }{ }^{28} ; \text { Kido et al. }^{29} \text {; } \\
\text { Lima-Gregio }^{32} \text {; Honjow e Isshiki }{ }^{33} ; \text { Kent et al. }{ }^{39}\end{array}$ \\
\hline Plosiva laríngea & $\begin{array}{l}\text { Videofluoroscopia } \\
\text { Nasoendoscopia }\end{array}$ & Witzel $^{18}$ \\
\hline Fricativa laríngea & $\begin{array}{c}\text { Videofluoroscopia } \\
\text { Nasoendoscopia } \\
\text { Espectrografia }\end{array}$ & Kawano et al. ${ }^{16,28}$ \\
\hline \multirow{2}{*}{ Africada laríngea } & Videofluoroscopia & Kawano et al. $^{28}$ \\
\hline & Nasoendoscopia & Kawano et al. ${ }^{16,28}$ \\
\hline \multirow{3}{*}{ Plosiva faríngea } & Radiografia & Trost $^{6}$; Phillips e Kent ${ }^{26}$ \\
\hline & Videofluoroscopia & Kawano et al. ${ }^{28} ;$ Honjow e Isshiki ${ }^{33}$ \\
\hline & Espectrografia & $\begin{array}{l}\text { Phillips e } \text { Kent }^{26} \text {; Kawano et } \text { al. }^{28} \text {; Honjow e } \\
\text { Isshiki }^{33}\end{array}$ \\
\hline \multirow[b]{2}{*}{ Fricativa faríngea } & Radiografia & Trost $^{6}$ \\
\hline & $\begin{array}{l}\text { Videofluoroscopia } \\
\text { Espectrografia }\end{array}$ & Weinberg e Horii ${ }^{40}$ \\
\hline \multirow{2}{*}{ Fricativa nasal posterior } & Radiografia & Trost $^{6}$ \\
\hline & Eletropalatrografia & Yamashita et al ${ }^{41}$ \\
\hline \multirow{3}{*}{ Plosiva dorso médio palatal } & Radiografia & Trost $^{6}$ \\
\hline & Eletropalatografia & $\begin{array}{l}\text { Gibbon e } \text { Crampin }^{11,42} \text {; Yamashita e Michi }{ }^{17} \text {; } \\
\text { Gibbon }^{43}\end{array}$ \\
\hline & Espectrografia & Sussman e Chapman ${ }^{44}$; Santelmann et al. ${ }^{45}$ \\
\hline
\end{tabular}

\section{Descrição dos tipos de AC}

A descrição dos tipos de AC é reportada considerando informações derivadas de avaliações clínicas e instrumentais.

\section{Oclusiva glotal (golpe de glote)}

A oclusiva glotal, mais conhecida como "golpe de glote", foi descrita na literatura por volta da década de $70^{4-14}$ e é considerado o tipo mais comum de AC produzido por sujeitos que apresentam FP e/ou DVF ${ }^{3,21,24,26}$. Esta AC é caracterizada por uma oclusão na região da laringe, em que "os articuladores usados são as pregas vocais (ao invés dos lábios e da língua) e o ponto articulatório é laríngeo (ao invés da cavidade oral). Ou seja, o ar é obstruído em uma região posterior à do ponto articulatório correto, sendo que a constrição do fluxo de ar ocorre antes que o mesmo atinja a válvula velofaríngea" (p.300) ${ }^{12}$.

A adução das pregas vocais, na produção da oclusiva glotal, está associada a um aumento da pressão aérea gerada na região subglótica, causando tensão na região mais baixa do trato vocal e, ainda, abertura e fechamento intensos das pregas vocais ${ }^{18}$. Além disso, acrescenta-se que a pressão e tensão envolvidas na oclusão glotal podem fazer com que as pregas ventriculares se desloquem medialmente, e até mesmo se toquem nessa região, indicando a presença de uma forma mais severa de obstrução na região laríngea ${ }^{4}$. A participação das pregas ventriculares durante a produção da oclusiva glotal foi confirmada por vários autores, a partir do uso de avaliações instrumentais ${ }^{18,28,29}$.

Com base em achados provenientes de avaliações nasofaringoscópicas, estudiosos ${ }^{27,29,30}$ descreveram dois sub-tipos de oclusivas glotais, que foram denominados por eles como sendo oclusiva glotal do Tipo 1 e Tipo 2. O primeiro tipo, considerado pelos autores como a oclusiva glotal típica, foi descrito como a adução das pregas vocais antes do início da vocalização e do ruído de soltura, parecido com uma vogal produzida com ataque vocal brusco, sem abdução das pregas vocais e sem participação da língua. Já o segundo tipo de oclusiva glotal foi 
descrito como adução pré-vocálica, seguida pela abdução das pregas vocais, o que corresponde à produção do ruído de soltura e nova adução para produzir a vogal seguinte, mostrando um padrão adução-abdução-adução. Por meio da videofluorosocopia, ainda observou-se que na oclusiva glotal do Tipo 1 a língua não entra em contato com o palato, enquanto que na oclusiva glotal do Tipo 2 algum tipo de movimento articulatório na cavidade oral foi observado para a maioria dos indivíduos, e comentaram que dados espectográficos auxiliaram na distinção desses subtipos de produções glotais ${ }^{36}$. Os mesmos autores ${ }^{30}$ sugerem que a oclusiva glotal Tipo 1 evolui para uma produção normal (não compensatória) via oclusiva glotal Tipo 2.

Um recente estudo nacional ${ }^{32}$ sobre a oclusiva glotal, utilizando espectrografia, também encontrou variabilidade. Foram analisadas 197 produções identificadas como oclusiva glotal, por no mínimo 3 de 5 juízes. Os resultados sugeriram que a presença de formantes de transição foi determinante para dúvida dos juízes, embora também tenha havido forte associação estatística para a presença de burst. Quando estes dois parâmetros estiveram ausentes na produção da oclusiva glotal, os juízes foram unânimes em afirmar a presença da AC.

De um modo geral, a oclusiva glotal, quando produzida como uma $\mathrm{AC}$ em associação à $\mathrm{FP}$ e/ ou DVF, ocorre em substituição para as consoantes que exigem maior pressão intra-oral, em especial, para as consoantes oclusivas e seus correlatos vozeados ${ }^{4,6,7,12,14,15,18,31}$. Embora tipicamente usada para substituir as consoantes oclusivas, esta $A C$ também pode ocorrer em substituição às fricativas ou africadas $3,7,20-21,25,31$, especialmente quando a criança ainda não adquiriu os fonemas fricativos em seu repertório linguístico ${ }^{31}$. Além disso, em casos considerados como mais severos, a oclusiva glotal também pode ocorrer em substituição aos líquidos e aos glides ${ }^{3,15}$.

A oclusiva glotal é auditivamente distinta de outros tipos de substituições ${ }^{7}$ e podem ser produzidas em combinação com outros movimentos articulatórios ${ }^{4,6,10,15,20}$, resultando em uma produção que se assemelha a produção correta dos sons alvos. Embora o ponto articulatório oral possa ser o correto (oclusão), a obstrução do fluxo aéreo ocorre na glote, isto é, o sujeito inicia a produção da oclusiva no nível da glote ${ }^{12,31}$ (ou seja, ocorre uma co-produção). Conforme relatos ${ }^{7}$, a oclusiva glotal pode ser co-produzida com qualquer uma destas consoantes de pressão: plosivas, fricativas e africadas.

A literatura reporta que é comum observar co-produção de oclusivas glotais por sujeitos que receberam fonoterapia, mas que não foram instruídos a direcionar o fluxo aéreo em combinação com o ponto articulatório desejado ${ }^{12}$. Além disso, na presença da oclusiva glotal, observa-se mínimo movimento velofaríngeo e, especialmente, das paredes laterais da faringe. Vários estudos investigaram a relação entre a produção glotal e o funcionamento velofaríngeo $0^{10,37,46}$, a partir de avaliação nasendoscópica e/ou videofluoroscópica. Os resultados destes estudos indicaram que os sujeitos apresentavam pouco movimento velofaríngeo na presença da oclusiva glotal, mas após breve período de terapia (com a instrução correta) observou-se melhora da movimentação velofaríngea para a maioria das crianças, ainda que a DVF continuasse presente.

\section{Plosiva laríngea}

As plosivas laríngeas, produzidas em substituição às consoantes oclusivas, são caracterizadas pelo movimento posterior de base da língua em direção à faringe, fazendo com que a epiglote entre em contato com a faringe durante a produção do som, resultando em um bloqueio momentâneo do fluxo aéreo ${ }^{18}$. Os achados videofluoroscópicos e nasendoscópicos, utilizados para descrever esse posicionamento dos articuladores na produção da plosiva laríngea, revelaram ainda que a laringe também parece se elevar de forma a favorecer temporariamente na obstrução do fluxo aéreo ${ }^{18}$.

\section{Fricativa laríngea}

As fricativas laríngeas, produzidas em substituição às consoantes fricativas ${ }^{4,12,15,16,26,28}$ são caracterizadas pelo movimento posterior de base da língua em direção à faringe, permitindo a aproximação da epiglote com a faringe, causando um estreitamento do fluxo de ar, gerando a fricção ${ }^{18}$. Também é reportado que a laringe eleva-se para auxiliar no estreitamento do fluxo aéreo ${ }^{16}$.

Desde em 1962 e em 1970, a literatura já se referia à fricativa laríngea em contraste com a fricativa faríngea, enfatizando que no caso da fricativa laríngea, as produções eram realizadas com friç̧ão aumentada entre as pregas vocais que se encontravam tensionadas excessivamente ${ }^{4}$. Devido à dificuldade em diferenciar as fricativas laríngeas e faríngeas, a partir exclusivamente da impressão auditiva, estas compensações são geralmente reportadas como fricativas faríngeas.

A partir de avaliações instrumentais, um pesquisador $^{16}$ documentou a fricativa laríngea por cinco sujeitos que produziam AC, sendo estas caracterizadas perceptivamente, por meio da avaliação perceptivo-auditiva, como fricativas faríngeas. Ao contrário do julgamento auditivo, as avaliações videofluoroscópicas e nasendoscópicas revelaram 
que estas fricativas eram produzidas ao nível da laringe, ao invés da faringe. Os achados espectrográficos também sugeriram a participação da laringe durante a produção desta compensação. Embora os pesquisadores tenham reportado o uso de fricativas laríngeas para cinco sujeitos, as informações detaIhadas foram reportadas somente para um destes sujeitos (20 anos, com fissura unilateral de lábio e palato, apresentando substituição de /s/ e /f/ por fricativa laríngea). As avaliações nasoendoscópicas e videofluoroscópicas confirmaram a presença de um ajuste laríngeo durante a produção da fricativa, caracterizado pelo movimento para cima, para frente e de adução das aritenóides em direção à epiglote. A análise de imagens de vídeo em slow motion mostrou ainda que a vogal seguida da fricativa laríngea era produzida enquanto as aritenóides (que estavam elevadas e aduzidas) voltavam a sua posição original. A partir destes achados os autores concluíram que a fricativa laríngea pode ser comumente encontrada entre as fricativas faríngeas, quando outros procedimentos são utilizados para complementar o julgamento perceptivo-auditivo ${ }^{16}$.

Resultados semelhantes foram reportados por outro autor ${ }^{28}$, que estudou 20 japoneses, dos quais 19 tiveram suas produções julgadas como fricativa faríngea, quando produziam, na realidade, fricativas laríngeas. Embora vários autores ${ }^{4,15,16,18,26,28}$ considerem a fricativa laríngea como um tipo de $A C$, outros ${ }^{7,24,31}$ sugerem que este tipo de produção parece ser uma variação da fricativa faríngea, justificando que o ponto articulatório é mais inferior.

\section{Africada laríngea}

As africadas laríngeas, produzidas em substituição às consoantes africadas, são caracterizadas pelo movimento posterior de base da língua em direção à faringe, fazendo com que a epiglote entre em contato breve com a faringe, causando constrição do fluxo aéreo para gerar a oclusão e, em seguida, a fricção ${ }^{18}$. Também é relatado que a laringe se eleva durante este tipo de produção ${ }^{16}$.

A partir de exames nasendoscópicos, estudiosos detectaram movimentos laríngeos atípicos para sons africados, os quais se assemelhavam àqueles reportados para as fricativas laríngeas ${ }^{16}$. Outros autores também reportaram que 16 dos 20 sujeitos que tiveram sua fala inicialmente julgada como fricativas faríngeas, apresentavam, na realidade, africadas laríngeas, quando avaliados por meio da nasoendoscopia e videofluoroscopia ${ }^{28}$.

\section{Plosiva faríngea}

As plosivas faríngeas, produzidas em substituição aos sons plosivos, são produzidas a partir do movimento do dorso da língua em direção à parede posterior da faringe ${ }^{6}$, sendo que o contato do dorso da língua na parede posterior da faringe provoca um aumento de pressão seguido de soltura súbita do fluxo aéreo ${ }^{18}$. A literatura reporta que a língua se move como um todo para trás, de forma a entrar em contato com a parede posterior da faringe, utilizando o fluxo aéreo que se encontra na faringe, antes do mesmo ser direcionado e escapar pela região velofaríngea. Além disso, um aumento da atividade laríngea pode ser notado na região do pescoço durante a produção da plosiva faríngea ${ }^{31}$.

Este tipo de $\mathrm{AC}$, em substituição à consoante velar $/ \mathrm{k} /$, foi identificada, por meio da cineradiografia e da espectografia, por pesquisadores que avaliaram a produção da fala de uma paciente de 28 anos, que apresentava fissura de lábio e palato reparada cirurgicamente ${ }^{33}$. Neste estudo, a plosiva faríngea foi julgada como uma produção perceptivamente aceitável desta consoante.

Estudos radiográficos demonstraram uma configuração convexa do dorso da língua em combinação com o abaixamento de sua ponta, associando estes movimentos como característicos da plosiva faríngea ${ }^{6}$. Tais estudos ainda evidenciaram que a oclusão pode ocorrer em diferentes regiões do tubo faríngeo (ou seja, mais próxima a região velofaríngea ou mais próxima a glote), sendo que essa variação no local em que se dá obstrução pode ser auditivamente identificada. De forma geral, observa-se que enquanto alguns autores ${ }^{33}$ consideraram a plosiva faríngea (produzida em japonês) como sendo uma substituição para o /k/, outro ${ }^{6}$ considera esta compensação (quando produzida no inglês) como perceptivamente e radiograficamente distinta de uma consoante plosiva, requerendo fonoterapia para adequação deste tipo de AC.

Em 1997, alguns estudiosos ${ }^{28}$, utilizando a videofluoroscopia, identificaram a produção de plosiva faríngea por 27 sujeitos que apresentavam FP e/ou DVF. Os autores reportaram variações no local de contato do dorso da língua na parede posterior da faringe durante a realização dessa AC, confirmando relatos prévios $^{6}$ sobre variabilidade em seu ponto articulatório, porém, diferente do que reportado em $1981^{6}$. Neste último foram identificados dois locais de obstrução, contudo no estudo de $1997^{28}$ foram descritos três pontos de contato do dorso da língua na parede posterior da faringe: (1) tipo orofaríngeo (observado em 8 sujeitos), (2) tipo oro e laringofaríngeo, em que tanto a epiglote quanto a base da língua entram em contato com a parede posterior da faringe (17 sujeitos) e (3) tipo epiglotal, em que a epiglote entra em contato com a parede posterior da faringe (2 sujeitos). Os autores ${ }^{28}$ colocam que o ponto da obstrução na faringe parece estar na dependência da idade do sujeito, sendo que na 
criança mais nova, a plosiva faríngea tende a ser produzida com a base da língua empurrando a epligote em direção à faringe, uma vez que a laringe está posicionada em uma região alta do pescoço e a epiglote encontra-se próxima ao palato mole. Já nas crianças mais velhas e nos adultos as plosivas faríngeas tendem a ser produzidas com a base da língua em contato com a parede posterior da faringe, em decorrência do crescimento da faringe na dimensão vertical. $O$ mesmo estudo ${ }^{28}$ ainda sugere que as plosivas faríngeas são influenciadas pelos sons, principalmente as vogais, que antecedem e/ou sucedem tal AC (informação também reportada no estudo de $1981^{6}$ ), além da velocidade de fala em que são produzidas.

Apesar de variações no ponto de contato entre o dorso da língua e a parede posterior da faringe serem relatadas na literatura, as plosivas faríngeas tem sido clinicamente julgadas como produções auditivamente distintas dos sons alvos $/ \mathrm{k} / \mathrm{e} / \mathrm{g} /$ e da oclusiva glotal ${ }^{1,7}$. Mais recentemente, uma autora ${ }^{24}$ enfatizou que a distinção entre a plosiva faríngea e a produção dos sons / $/ \mathrm{k}$ e /g/ pode não ser uma tarefa fácil, e que devido às dificuldades impostas para a sua produção não há co-produção ${ }^{1,7,24}$.

\section{Fricativa faríngea}

A fricativa faríngea, em associação à presença de FP e/ou DVF, foi descrita em substituição às consoantes fricativas ${ }^{4,14,15}$ (e, em especial, as sibilitantes) e às africadas, vozeadas e não vozeadas $^{4,7,12,15,25,31}$. As fricativas faríngeas podem ser co-produzidas com o ponto articulatório do fonema alvo $^{6-7,10}$. São produzidas quando o dorso da língua se aproxima da parede posterior da faringe a fim de gerar constrição do fluxo de ar, resultando em friç̧ão ${ }^{6,18,20,21,24}$. A fricção ocorre abaixo da válvula velofaríngea, comprometendo a movimentação das estruturas velofaríngeas durante a produção desta compensação ${ }^{37}$.

Em um estudo 6 baseado em visão radiográfica (lateral), verificou-se uma configuração mais posteriorizada e côncava do dorso lingual durante a produção das fricativas faríngeas. Achados clínicos sugerem que o local de constrição do trato vocal pode ocorrer em uma região mais alta ou mais baixa da faringe ${ }^{7}$. Também é reportado que existem dois tipos de fricativas faríngeas, de acordo com a região em que se observa a constrição na faringe ${ }^{21}$. $\mathrm{Na}$ fricativa faríngea em que a constrição ocorre em uma região mais alta da faringe, o ruído resultante desta constrição é menos intenso. Já na fricativa localizada em um ponto mais baixo da faringe, observa-se maior tensão faríngea e maior rebaixamento de dorso da língua.

\section{Africada faríngea}

As africadas faríngeas são definidas como sons produzidos simultaneamente com a válvula glótica e linguofaríngea. Essas compensações envolvem a combinação da oclusiva glotal e da fricativa faríngea ${ }^{7}$. Nesta $A C$, o dorso da língua se move posteriormente entrando em contato com a faringe, a fim de criar constrição do fluxo de ar, gerando oclusão e, em seguida, a friç̧ão ${ }^{18}$. Um aumento na atividade faríngea pode ser visualmente obervado durante a produção da africada faríngea ${ }^{31}$, sendo que uma configuração mais posteriorizada e côncava do dorso lingual é observada durante a produção desta $\mathrm{AC}^{7}$.

As africadas faríngeas são comumente usadas em substituição às africadas orais e não são co-produzidas com o ponto articulatório do fonema alvo ${ }^{7,31}$. Além disso, pode ser difícil distinguir auditivamente a africada faríngea da fricativa faríngea, especialmente durante a conversa espontânea ${ }^{31}$.

\section{Fricativa velar}

A fricativa velar EM associação à presença de FP e/ou DVF $3,6,7,15,20,31$ é produzida pela fricção realizada a partir do contato do dorso da língua e do palato mole, próxima ao local em que ocorre a produção do $/ \mathrm{k} /$ e $/ \mathrm{g} /{ }^{6}$. Conforme apontado na literatura, a produção de fricção característica da fricativa velar é resultado de um pequeno espaço criado em decorrência da elevação do dorso da língua que se posiciona abaixo da região velar ${ }^{31}$. As fricativas velares são percebidas como distorções de /k/ e /g/ devido à uma perda da qualidade da oclusão? .

A fricativa velar em substituição às consoantes fricativas é reportada por vários autores ${ }^{6,7,15,31}$, sendo que são mais comumente usadas em substituição à fricativas sibilantes quando associada à FP e/ou DVF', não ocorrendo co-produção com outros articuladores ${ }^{7}$. Vale ressaltar que, em 2006, alguns autore $^{1}$ passam a caracterizar a fricativa velar (anteriormente considerada como uma AC) como um tipo de "produção oral posteriorizada".

\section{Fricativa nasal}

$\mathrm{Na}$ fricativa nasal, também chamada fricativa nasal ativa ${ }^{8,35}$, observa-se emissão de ar nasal sem turbulência, com oclusão oral completa, sendo que todo o fluxo aéreo é direcionado para a cavidade nasal $^{1}$. Esta AC é usada predominantemente em substituição às consoantes fricativas alveolares $e$ palatais ${ }^{1}$. A literatura descreve um tipo de produção caracterizado pela elevação de língua e direcionamento de todo o fluxo aéreo pelo nariz durante a tentativa de produção das consoantes fricativas e, em particular, do /s/, denominando este tipo de produção de de sigmatismo nasal ${ }^{21}$. Note-se que tal 
descrição assemelha-se àquela da fricativa nasal, conforme apresentada por outros autores ${ }^{8,35}$. A literatura ${ }^{24}$ reporta uma diferenciação na produção da fricativa nasal, por meio da constrição na região nasofaríngea, em que a friç̧ão ocorre com a aproximação do véu em direção à parede posterior da faringe. Tal descrição, no entanto, parece estar mais próxima do que a literatura denomina como fricativa nasal posterior.

\section{Fricativa nasal posterior}

A fricativa nasal posterior é descrita em substituição às consoantes fricativas 6 . De forma geral, a produção desta AC ocorre quando o palato mole se aproxima da parede posterior da faríngea, mas não permite fechamento velofaríngeo completo. Com base em estudos radiográficos ${ }^{1,6}$, verificou-se uma variabilidade nos movimentos articulatórios: enquanto a fricção, em alguns casos, é gerada ao nível da região velofaríngea, em outros, há elevação e posteriorização da língua, o que indica assistência da língua para compensar a DVF, especialmente em casos de fissura submucosa ou alterações de neurogênicas.

Embora algum grau de emissão de ar nasal esteja, geralmente, associado à fricativa nasal posterior, a emissão de ar nasal resultante desta AC é perceptualmente distinta da emissão de ar nasal não associada à $A C$, sendo observados diferentes locais de turbulência para estes dois fenômenos. No caso de bloqueio nasal ou, ainda, de retalho faríngeo obtrusivo, a fricativa nasal posterior pode ser o único fenômeno a ser ouvido ${ }^{6}$. A fricativa nasal posterior também é considerada perceptivamente distinta das fricativas velares e faríngeas ${ }^{7}$. Esta AC é comumente observada em associação à emissão de ar nasal aprendida, que ocorre em fonemas específicos ${ }^{1}$.

Com base em medidas eletropalatográficas e espectrográficas, estudiosos ${ }^{17}$ observaram contatos de língua com a nasofaringe durante a produção da fala de crianças com FP, o que levou à associação destes achados com a fricativa nasal posterior descrita previamente ${ }^{6}$. Contudo, os mesmos contatos articulatórios foram observados durante a produção de sons fricativos e, ainda, de sons plosivos e africados de crianças que não apresentavam DVF e/ou fístula ${ }^{17}$.

\section{Africada nasal posterior}

A africada nasal ocorre em substituição às consoantes africadas ${ }^{7,18}$. Nestas produções, o dorso da língua e o palato mole são normalmente posicionados para gerar plosão e friç̧ão na válvula velofaríngea. A emissão de ar nasal audível está sempre associada à esta AC.

\section{Plosiva dorso médio palatal}

A literatura descreve a plosiva dorso-médiopalatal como uma produção que ocorre com o contato da parte média da língua com o palato duro (no local aproximado onde o glide /j/ seria produzido) $)^{6}$. Quando associada com DVF ou fístula no palato, essa AC é observada em substituição aos sons $/ \mathrm{t} / \mathrm{l} / \mathrm{d} / \mathrm{l} / \mathrm{k} /$ ou $/ \mathrm{g} /$. Mais especificamente, em um estudo radiográfico ${ }^{6}$, observou-se a elevação da região média da língua em contato com o palato duro, estando a ponta da língua para baixo durante a produção. A diferenciação auditiva entre /t/ e / $/ \mathrm{k} /$ ou entre $/ \mathrm{d} / \mathrm{e} / \mathrm{g} /$ fica comprometida, pois o dorso da língua se posiciona de maneira semelhante para ambos os alvos ${ }^{6}$.

Vários autores também reportam a plosiva dorso médio palatal na fala de sujeitos com FP e/ ou DVF'12,18,21,24,31. A literatura ${ }^{41}$ aponta que esta $\mathrm{AC}$ somente pode estar associada à DVF, quando em substituição aos sons alveolares, o que resulta em um padrão de posteriorização da língua. Outra autora $^{18}$ relata que a plosiva dorso médio palatal é identificada na presença de fístula de palato, sugerindo uma tentativa de oclusão da fístula, com a língua, enquanto ocorre a produção do som. Vários outros autores ${ }^{12,18,31,37}$ comentam que esta $A C$ não está associada à DVF, mas à presença de fístula (anterior) de palato ou, ainda, às alterações oclusais em sujeitos com $\mathrm{FP}^{12,31}$.

Alguns autores discutem a origem da plosiva dorso médio palatal a partir das discussões do termo $A C$ da literatura ${ }^{3}$. Mais recentemente, esta $A C$ foi resumida como: (a) resultante de padrões estabelecidos no período de aquisição fonológica quando a FP ainda está aberta; (b) resultante de um padrão de postura de língua estabelecido numa tentativa de ocluir a fístula de palato e (c) observada como produções transitórias em crianças sem história de FP e/ou DVF3. Vários estudos buscaram um melhor entendimento deste tipo de AC, a partir do uso da análise acústica ${ }^{44,45}$ e da eletropalatografia ${ }^{41,42}$. Em um destes estudos ${ }^{42}$, foi observado que a plosiva dorso médio palatal em substituição aos sons alveolares e velares, foi julgada de forma idêntica na avaliação perceptivo-auditiva. No entanto, os achados obtidos pela eletropalatografia evidenciaram que o ponto articulatório para os sons alveolares era realizado mais anteriorimente do que para os sons velares. Ainda foi observada fricção lateral, sendo possível quantificar, por meio de medidas instrumentais, o tempo de fricção lateral associado ao período de aspiração durante a produção desta AC. 


\section{Fricativa dorso médio palatal}

A fricativa dorso médio palatal foi descrita como resultante da aproximação do dorso médio da língua no palato duro, a fim de gerar fricção próximo ao local onde o glide /j/ seria produzido ${ }^{18}$. Esta $A C$, também chamada de fricativa palatal, foi reportada previamente ${ }^{9,26}$ como uma produção mais posteriorizada para as consoantes alveolares e palatais, mas não tão posteriormente quanto as velares.

A fricativa dorso-médio-palatal é comumente identificada na presença de fístula no palato, indicando uma tentativa de se ocluir a fístula com a língua durante a sua produção ${ }^{18}$. Por outro lado, alguns autores ${ }^{17}$, baseados em medidas eletropalatográficas e espectrográficas, reportaram achados sugestivos de contato do dorso médio da língua no palato duro para a produção de fricativas em crianças com FP sem fístula de palato. Outros estudiosos $^{37}$ observaram o uso de fricativa dorso médio palatal, em consoantes fricativas sibilantes, por crianças com fístula anterior de palato, e sugeriram que a fonoterapia para este tipo de AC pode apresentar resultados mais satisfatóriso após o fechamento da fístula.

\section{Africada dorso médio palatal}

A africada dorso-médio-palatal foi descrita como resultado do contato e aproximação da região do dorso médio da língua no palato duro, a fim de gerar fricção próxima ao local onde o glide /j/ seria produzido ${ }^{18}$. A fricativa dorso-médio-palatal tem sido quase sempre identificada com a presença de fístula no palato, indicando uma tentativa de se ocluir a fístula durante a sua produção ${ }^{18}$.

\section{Avaliação clínica e instrumental na identificação das AC: implicações e contribuições}

A literatura consultada revela uma grande preocupação na descrição das AC, por meio de métodos subjetivos ou objetivos. No entanto, a prática clínica tem sido baseada, fundamentalmente, em impressões subjetivas sobre os movimentos articulatórios e o resultado auditivo das AC. Ou seja, a caracterização dos movimentos articulatórios envolvidos nas AC é guiada, predominantemente, pelo julgamento perceptivo (auditivo e visual) dos avaliadores.

Tal julgamento não é uma tarefa fácil, mesmo para profissionais com vasta experiência na avaliação e no tratamento das alterações de fala decorrentes da FP e/ou DVF, ainda que eles se apóiem na transcrição das produções de fala para estabelecer a conduta e documentar o processo terapêutico. Da mesma forma, essa prática pode ser ainda mais difícil, principalmente para profissio- nais sem experiência na avaliação e no tratamento da fala de crianças com FP e/ou DVF ${ }^{47,48}$.

Vários estudiosos ${ }^{45,47,48}$ apontaram dificuldades na análise perceptivo-auditiva para a identificação das alterações de fala, incluindo as AC, associada à FP e/ou DVF. Por exemplo, um estudo ${ }^{47}$ observou maior concordância na identificação das AC por profissionais com experiência na avaliação de sujeitos com FP, quando comparadas àquela obtida por profissionais sem experiência, quando a transcrição fonética foi utilizada. Embora, neste estudo, para ambos os profissionais (com e sem experiência), a concordância inter-juízes foi baixa, o que levou os autores a concluírem sobre a necessidade de treinamento específico para os avaliadores que atuam com sujeitos que apresentam AC. Outro estudo ${ }^{49}$ também encontrou concordância baixa entre juízes experientes na identificação de produções atípicas e atribuiu este achado à falta de treinamento suficiente para os avaliadores. De forma geral, os dados obtidos nestes estudos evidenciam a necessidade de treinamento específico para a identificação das produções compensatórias, mesmo para avaliadores com experiência no atendimento de sujeitos com AC. Nesse sentido, verifica-se, principalmente em âmbito nacional, a necessidade da elaboração de materiais específicos que contemplem os diferentes tipos de AC, os quais forneçam descrições detalhadas, associadas aos recursos audiovisuais, a fim de contribuir para um maior conhecimento de fonoaudiólogos. A apresentação destes materiais pode ser beneficiada pela Telessaúde ${ }^{50}$, que é entendida como uma ferramenta de ensino que permite o desenvolvimento de programas educacionais para atualização e treinamento de profissionais, oferecendo, portanto, capacitação a partir de programas especializados, conforme já propostos em outras áreas da Fonoaudiologia ${ }^{51}$.

Outro aspecto amplamente discutido na literatura especializada em $\mathrm{FP}^{52}$ é a necessidade do uso de procedimentos clínicos padronizados, que favoreçam a coleta e a análise de informações perceptivas da fala de sujeitos com FP, incluindo as $\mathrm{AC}^{53,54}$. Além disso, há a necessidade de se utilizar parâmetros perceptivos universais para reportar a fala destes sujeitos, com o objetivo de favorecer a obtenção de dados mais consistentes, que permitem uma uniformidade nos resultados encontrados pelos avaliadores e centros de pesquisa.

Com relação aos procedimentos padronizados (para fins clínicos e de pesquisa), é pertinente que se desenvolva, em âmbito nacional, um procedimento que contemple as consoantes de interesse em suas diferentes posições silábicas, no nível da palavra e em textos, e, também, que contemple 
níveis mais elaborados do discurso, assim como acontece na conversa espontânea. Ainda, é importante que se estabeleçam critérios comuns para o armazenamento das informações (gravações em áudio, áudio e vídeo), para que elas possam ser utilizadas no julgamento dos avaliadores, a fim de minimizar problemas relatados na literatura ${ }^{55}$. Essa prática também pode beneficiar o paciente em seu processo terapêutico, favorecendo não somente a identificação das AC por ele produzidas, mas, sobretudo, as modificações dos ajustes articulatórios durante a fonoterapia.

A literatura consultada sugere que uma identificação mais precisa das $\mathrm{AC}$ pode ser obtida quando a avaliação perceptiva destas produções está associada às informações derivadas da avaliação acústica (espectrografia) ${ }^{32,45,47,56}$ e da eletropalatografia ${ }^{42,43}$. Por meio destas avaliações, pode-se confirmar e, ainda, acrescentar informações sobre possíveis ajustes articulatórios envolvidos na produção das AC ou, até mesmo, rever impressões subjetivas sobre estas produções ${ }^{42,57}$. Além dessas técnicas, informações importantes sobre os ajustes articulatórios associados à fala de sujeitos com FP e DVF podem ser derivados de outras avaliações instrumentais, como a nasoendoscopia ${ }^{18}$, videofluoroscopia ${ }^{18}$ e articulografia ${ }^{58}$. Há relatos na literatura ${ }^{59}$, de que as avaliações nasoendoscópica e acústica também podem colaborar no monitoramento do processo terapêutico de sujeitos com FP, ainda, podem ser utilizadas como biofeedback para o paciente. Ainda há relatos na literatura internacional ${ }^{60}$ sobre as vantagens do uso da ultrasonografia como um recurso para favorecer feedback visual no processo terapêutico de crianças que apresentam fala desviante, uma vez que esta avaliação permite mostrar ao paciente a posição e a forma que a língua assume na cavidade oral. Neste sentido, sujeitos com FP também poderiam se beneficiar desta técnica no processo terapêutico direcionado para as AC e, particularmente, para aquelas que envolvem regiões mais anteriores da cavidade oral, como é o caso das compensações envolvendo a região dorso médio palatal.

Embora as contribuições destes instrumentos sejam descritas na literatura internacional, eles ainda são pouco utilizados no Brasil para fins clínicos e de pesquisa. As informações obtidas da avaliação instrumental são necessárias para um melhor entendimento sobre a produção da fala associada à FP/ DVF, uma vez que podem fornecer parâmetros que sinalizem as diferenças entre a produção normal e alterada (AC), bem como entre os diferentes tipos de $A C$, o que nem sempre é possível somente a partir da avaliação perceptivo-auditiva. Além disso, estas avaliações podem revelar a presença de ajustes articulatórios que não são percebidos auditivamente e, portanto, podem favorecer o estabelecimento do planejamento terapêutico.

\section{CONCLUSÃO}

A literatura consultada contribuiu para um aprofundamento sobre AC na medida em que informações históricas e metodológicas foram apresentadas. Tais informações justificam a terminologia utilizada por diferentes autores, bem como apresentam uma descrição detalhada dos movimentos articulatórios envolvidos em cada tipo de AC, a partir de informações derivadas da avaliação subjetiva e instrumental. A literatura traz, ainda, informações sobre as implicações da avaliação subjetiva como único recurso para a identificação das $A C$ e as contribuições da avaliação objetiva para um maior entendimento destas produções, de forma a favorecer a identificação, bem como o planejamento terapêutico. As AC merecem a atenção de clínicos e pesquisadores que atuam no Brasil, já que estas alterações ainda são encontradas com grande freqüência em crianças e, até mesmo, em adultos com FP e/ DVF, o que compromete a qualidade de vida destes sujeitos. Cabe aos profissionais fonoaudiólogos o aprofundamento de conhecimentos sobre estes tipos de produções para favorecer a inteligibilidade de fala de sujeitos com FP e/ou DVF que desenvolveram as $A C$, bem como é de responsabilidade destes profissionais o estabelecimento de programas preventivos que favoreçam a aquisição fonológica sem o desenvolvimento dessas compensações. 


\section{ABSTRACT}

Background: compensatory articulation in cleft lip and palate. Purpose: to contribute with information regarding the types of compensatory articulation described in the literature and discuss the implications and contributions of clinical and instrumental evaluation of these speech productions. Conclusion: compensatory articulation deserves attention from Brazilian physicians and researchers, since that these productions occur in children and adults with cleft palate and velopharyngeal dysfunction, compromising their speech intelligibility and consequently quality of their lives. Speech-language pathologists need to improve knowledge regarding different types of compensatory articulation and also on the procedures for evaluating these productions, in order to settle preventive programs that favor phonological acquisition in children with cleft palate without developing compensatory articulation.

\section{KEYWORDS: Cleft Palate; Speech; Speech Disorders; Articulation Disorders}

\section{REFERÊNCIAS}

1. Peterson-Falzone SJ, Trost-Cardamone JE, Karnell MP, Hardin-Jones MA. The clinician's guide to treating cleft palate speech. St. Louis: Mosby; 2006.

2. Bzoch KR. Measurement and assessment of categorical aspects of cleft palate speech. In: Bzoch KR (ed). Communicative disorders related to cleft lip and palate. Boston: Little, Brown; 1979. p. 161-91. 3. Peterson-Falzone SJ, Hardin-Jones MA, Karnell MP. Communication disorders associated with cleft palate. Cleft palate speech. 3rd ed. St. Louis: Mosby; 2001. p. 162-98.

4. Morley ME. Cleft palate and speech. 7th ed. Baltimore: Williams \& Wilkins; 1970.

5. Bzoch KR. A battery of clinical perceptual tests, techniques, and observations for the reliable clinical assessment, evaluation, and management of 11 categoriasl aspects of cleft palate speech disorders. In: Bzoch KR (ed). Communicative disorders related to cleft lip and palate. 5th ed. Austin: Pro-Ed; 2004. p. 375-462.

6. Trost JE. Articulatory additions to the classical description of the speech of persons with cleft palate. Cleft Palate J. 1981;18:193-203.

7. Trost-Cardamone JE. Diagnosis of specific cleft palate speech error patterns for planning therapy or physical management needs. In: Bzoch KR (ed). Communicative disorders related to cleft lip and palate. 4th ed. Austin: Pro-Ed; 1997. p. 313-30.

8. Harding A, Grunwell P. Active versus passive cleft type speech characteristics. Int J Language Communication Dis. 1998;33:329-52.

9. McWilliams BJ, Morris HL, Shelton R L. Cleft palate speech. Philadelphia: Decker; 1990.

10. Henningsson GE, Isberg AM. Velopharyngeal movement patterns in patients alternating between oral and glottal articulation: a clinical and cineradiographical study. Cleft Palate J. 1986; 23:1-9.

11. Gibbon FE, Crampin L. Labial-lingual double articulations in speakers with cleft palate. Cleft Palate J. 2002; 39(1):40-9.

12. Golding-Kushner K. Treatment of articulation and resonance disorders associated with cleft palate and VPI. In: Shprintzen RJ, Bardach J. Cleft palate speech management: a multidisciplinary approach. Mosby: St. Louis; 1995. p. 327-51.

13. Lawrence C, Phillips BJ. A telefluoroscopic study of lingual contacts made by person with cleft palate. Cleft Palate J. 1975;12: 85-94.

14. Morris HL. Abnormal articulation patterns. In: Bzoch KR (ed). Communicative disorders related to cleft lip and palate. Boston: Little, Brown; 1971.

15. Bzoch KR. Categorical aspects of cleft palate speech. In: Grabb, WC, Rosenstein SW, Bzoch, KR (ed). Cleft lip and palate: surgical, dental, and speech aspects. Boston: Little, Brown; 1971. p. 713-33.

16. Kawano M, Isshiki N, Harita $Y$, Tanokuchi F. Laryngeal fricative in cleft palate speech. Acta Otolaryngol Suppl. 1985; 419:180-8.

17. Yamashita Y, Michi K. Misaticulation caused by abnormal lingua-palatal contact in patient with cleft palate and adequate velopharyngeal function. Cleft Palate J. 1991; 28(4):360-8.

18. Witzel MA. Communicative impairment associated with clefting. In: Sphrintzen RJ, Bardach $\mathrm{J}(\mathrm{ed})$. Cleft palate speech management. Mosby: St. Louis; 1995.

19. Mattos ALG. Padrão de articulação compensatória de indivíduos com inadequação velofaríngea: revisão de literatura [monografia]. Rio de Janeiro (RJ): Instituto de Reabilitação; 1990.

20. Vicente MCZ, Buchala RG. Atualização da terminologia de distúrbios articulatórios encontrados 
em falantes portadores de fissura de lábio e palato. Dist Comum. 1991; 4(2):147-52.

21. Altmann EBC, Ramos, ALNF, Khoury, RBF. Avaliação fonoaudiológica. In: Altmann EBC. Fissuras Labiopalatinas. Carapicuíba: Pró-Fono; 1997. p.325-66.

22. Jesus MSV, Penido FA, Valente P. Avaliações fonoaudiológicas clínica e instrumental em indivíduos com fissura labiopalatina. In: Jesus MSV, Di Ninno CQMS (ed). Fissura Labiopalatinas: fundamentos para a prática fonoaudiológica. São Paulo: Roca; 2009. p. 57-75.

23. Genaro KF, Yamashita RP, Trindade, IEK. Avaliação clínica e instrumental na fissura labiopalatina. In: Ferreira LP, Befi-Lopes DM, Limongi SCO (ed). Tratado de Fonoaudiologia. São Paulo: Roca; 2004. p.456-77.

24. Hanayama EM. Distúrbios da comunicação nos pacientes com sequela de fissura labiopalatina. Rev Bras Cir Craniomaxilofac. 2009; 12(3):118-24.

25. Golding-Kushner KJ. Therapy techniques for cleft palate speech and related disorders. San Diego: Singular; 2001.

26. Philips BJ, Kent RD. Acoustic-phonetic descriptions of speech production in speakers with cleft palate and other velopharyngeal disorders. In: Lass NJ (ed). Speech and language: advances in basic research and practice. Orlando: Academic Press.; 1984; 11:113-68.

27. Kawano M, Honjo I, Kojima H, Kurata F, Tanokuchi F, Kido N. Laryngeal constrictions on glottal stop in cleft palate speech. Studia Phonologica. 1991; XXV:7-13.

28. Kawano M. Isshiki N, Honjo I, Kojima $\mathrm{H}$, Kurata K, Tanokuchi F. Recent progress in treating patients with cleft palate. Folia Phoniatr Logo. 1997; 49:117-38.

29. Kido N, Kawano M, Tanochushi F, Fujiwara $Y$, Honjo I, Kojima H. Glottal stop in cleft palate speech. Studia Phonologica 1992; XXVI:34-41.

30. Kido N, Kawano M, Tanokuchi F, Fujiwara Y, Kurata K, Kojima H, Honjo I. Glottal stop in cleft palate speech (2nd report): dynamic alterations of laryngeal movement during production of voiceless stop CV syllables. Studia Phonologica 1993; XXVII:33-41.

31. Kummer AW. Velopharyngeal dysfunction and resonance disorders. In: Kummer AW (ed). Cleft palate \& craniofacial anomalies: effects on speech and resonance. San Diego: Singular; 2001. p.145-76.

32. Lima-Gregio AM. Oclusiva glotal e laringalização em sujeitos com fissura palatina: um estudo segundo abordagem dinamicista [Tese]. Campinas (SP): Universidade Estadual de Campinas; 2011.
33. Honjow I, Isshiki N. Pharyngeal stop in cleft palate speech. Folia Phoniatr. 1971; 23:325-54.

34. Peterson-Falzone SJ, Graham MS. Phonemespecific nasal emission in children with and without physical anomalies of the velopharyngeal mechanism. J Speech Hear Disord. 1990; 55:132-9. 35. Sell D, Harding A, Grunwell P. GOS.SP.ASS. 98: an assessment for speech disorders associated with cleft palate and/or velopharyngeal dysfunction (revised). Int Lang Commun Disord. 1999; 34:17-33. 36. Peterson-Falzone SJ. Speech characteristics: updating clinical decisions. Semin Speech Lang. 1986; 7:269-95.

37. Hoch L, Golding-Kushner K, Siegel-Sadewitz VL, Shprintzen RJ. Speech therapy. Semin Speech Lang. 1986; 7:313-26.

38. Warren DW. Compensatory speech behaviors in cleft palate: A regulation/control phenomenon. Cleft Palate J. 1986; 23:251-6.

39. Kent RE, Liss JM, Phillips BJ. Acoustic analysis of velopharyngeal dysfunction in speech. In: Bzoch KR (ed). Communication disorders related to cleft lip and palate. 4th ed. Austin: Pro-Ed; 1999.

40. Weinberg B, Horii Y. Acoustic features of pharyngeal /s/ fricatives produced by speakers with cleft palate. Cleft Palate J. 1975; 12:12-6.

41. Yamashita Y, Michi K, Imai S, Suzuki N, Yoshida $\mathrm{H}$. Eletropalatographic investigation of abnormal lingual-palatal contact patterns in cleft palate patients. Clin Linguist Phonet. 1992;6(3):201-17.

42. Gibbon FE, Crampin L. An electropalatographic investigation of middorsum palatal stops in an adult with repaired cleft palate. Cleft Palate J. 2001;38:96-105.

43. Gibbon FE. Abnormal patterns of tongue-palate contact in the speech of individuals with cleft palate. Cleft Palate J. 2004;18(4-5):285-311.

44. Sussman J, Chapman K. Acoustic analysis of speech: a research study. Presented at Craniofacial Update: Interdisicplinary Advances. Buffalo: Craniofacial Center of Western New York; 1995.

45. Santelman L, Sussman J, Chapman K. Perception of middorsum palatal stops from the speech of three children with repaired cleft palate. Cleft Palate J. 1999;36: 233-42.

46. Ysunza, A. Pamplona, C. Toledo, E. Change in velopharyngeal valving after speech therapy in cleft palate patients: a videonasopharyngsocopic and multi-view videofluoroscopic study. Int J Pediatr Otorhi. 1992;24:45-54.

47. Gooch J, Hardin-Jones M, Chapman K, TrostCardamone J, Sussman J. Reliability of listener judgments of compensatory articulations. Cleft Palate J. 2001;38:59-67. 
48. Howard SJ, Heselwood BC. Learning and teaching phonetic transcription for clinical purposes. Clin Linguist Phonet. 2002;16:371-401.

49. Brunnegard, K.; Lohmander, A. A crosssectional study in 10-year-old children with cleft palate: results and issues of rater reliability. Cleft Palate J. 2007;44:33-44.

50. Wen CL. Cadeia Produtiva de Saúde: uma concepção mais ampla da Telemedicina e Telessaúde. RevTelem Telessaude. 2006;2(2):8-10. 51. Spinardi ACP, Blasca WQ, Wen CL, Maximino LP. Telefonoaudiologia: ciência e tecnologia em saúde. Pró-Fono R Atual Cient. 2009;21(3):249-54. 52. Hennigsson G, Kuehn DP, Sell D, Sweeney $\mathrm{T}$, Trost-Cardamone JE, Whitehill TL. Universal parameters for reporting speech outcomes in individuals with cleft palate. Cleft Palate J. 2008;45(1):1-17.

53. Lohmander A, Olson M. Methodology for perceptual assessment of speech in patients with cleft palate: a critical review of the literature. Cleft Palate J. 2004;41(1):64-70.

54. Sell D. Issues in perceptual speech analysis in cleft palate and related disorders: a review. Int $\mathrm{J}$ Lang Com Dis. 2005;40(2):103-21.
55. Sell D, John A, Harding-Bell A, Sweeney T, Hegarty F, Freeman J. Cleft audit protocol for speech (CAPS-A): a comprehensive training package for speech analysis. Int J Lang Comm Dis. 2008;1-20.

56. Sharin K. Remarks on the speech of Arabicspeaking children with cleft palate. California Linguistic Notes. 2002;17(1):1-10.

57. Gibbon FE, Lee A, Yuen I, Crampin L. Clicks Produced as Compensatory Articulations in Two Adolescents With Velocardiofacial Syndrome. Cleft Palate J. 2008;45(4):381-92.

58. Marino VCC, Williams WN, Wharton PW, Paulk MF, Dutka-Souza JCR, Schulz GM. Immediate and sustained changes in tongue movement with an experimental palatal "fistula": a case study. Cleft Palate J. 2005; 42(3):286-96.

59. Howard S. Compensatory articulatory behaviors in adolescents with cleft palate: comparing the perceptual and instrumental evidence. Clin Linguist Phonet. 2004;18(4-5):313-40.

60. Bernhardt B, Gick B, Bacsfalvi P, AdlerBock M. Ultrasound in speech therapy with adolescents and adults. Clin Linguist Phonet. Special Issue: Ultrasound Imaging of the Tongue. 2005;19(6):605-17.
http://dx.doi.org/10.1590/S1516-18462011005000077

RECEBIDO EM: 18/02/2011

ACEITO EM: 18/03/2011

Endereço para correspondência:

Viviane Cristina de Castro Marino

Av. Higino Muzzi Filho, 737

Marília - SP

CEP 17525-900

E-mail: vivianemarino2@yahoo.com.br 